\title{
Le statut problématique des Études francophones en Allemagne
}

\author{
Timo Obergöker \\ Johannes Gutenberg Universität Mainz
}

\begin{abstract}
Parler du paysage scientifique dans son propre pays soulève un certain nombre de difficultés. D’abord des difficultés pratiques qui se posent à tout chercheur qui critique son propre champ de recherche et qui va ainsi éventuellement blesser les susceptibilités de certains collègues; ensuite, problème herméneutique, la difficulté du manque de distance qui sépare le chercheur de son objet d'étude ; et finalement le fait que les Études Francophones et les pays de langue allemande constituent tous les deux des entités complexes, si bien que l'écueil de la simplification outrancière ne pourra sans doute pas toujours être évité.
\end{abstract}

Afin d'aborder l'émergence des Études Francophones en Allemagne et afin de prendre la mesure des problèmes qu'elle a soulevés, il convient dans un premier temps de parler de l'organisation de la «Romanistique » au sein de l'Université allemande, suite à quoi on va aborder le statut très complexe du français en Allemagne. Il convient, à ce propos, également d'aborder le statut particulier des relations franco-allemandes. Ce n'est qu'après avoir présenté le cadre institutionnel dans lequel évoluent les Études Françaises en Allemagne que l'on pourra se consacrer aux Études Francophones proprement dites. Si effectivement, à l'heure qu'il est, les Études Postcoloniales et les Études Francophones sont en plein essor en Allemagne les résistances institutionnelles étaient importantes. C'est de l'émergence des Études Francophones en Allemagne que parlera ce texte.

\section{1) La Romanistique - une passion tout allemande}

L'histoire de la Romanistique allemande qui remonte au XIXe siècle est compliquée. Dans ses débuts, elle était étroitement liée à la philologie de l'édition et se consacrait essentiellement à l'édition des textes médiévaux domaine dans lequel elle obtint effectivement des résultats étonnants. Il convient néanmoins de tenir compte du fait que la naissance de la Romanistique allemande (le premier institut de langues romanes fut fondée à Bonn en 1830) coïncide avec l'avènement du nationalisme allemand qui, lui, est considéré comme une contre-réaction par rapport aux guerres napoléoniennes. On comprendra facilement que cette situation est pour le moins alambiquée et crée un imaginaire complexe (Christmann).

Puisque l'université allemande, institution conservatrice et traditionnaliste s'il en est, continue depuis le XIXe siècle à envisager les langues romanes comme une unité, le français fait partie d'un département de langues romanes au sein duquel il est la langue dominante, un rôle qui lui sera d'ailleurs de plus en plus contesté par l'espagnol. Eu égard à cette unité, les romanistes allemands ont habituellement une double spécialisation, habituellement le français plus l'italien ou l'espagnol ou l'espagnol et le portugais. Rares sont les professeurs allemands dont la recherche et l'enseignement se limitent à une seule langue. Pour le français, il en existe et ces professeurs jouent alors souvent un rôle important pour les Études Francophones. Par ailleurs, le système 
universitaire allemand est organisé autour du professeur titulaire et ne connait guère les professeurs-assistants ou associés. En fait le personnel enseignant non titulaire de l' « habilitation » est « en voie de qualification » c'est-à-dire qu'il est ou bien en train de préparer un doctorat ou l'habilitation ou qu'il est réduit à des postes subalternes à forte charge de cours pour ce qui est des rares postes à durée illimitée en dehors du professorat. Il se trouve toutefois que cette catégorie de subalternes domine surtout pendant les trois premières années et que les professeurs titulaires sont relativement peu nombreux au sein des départements. Afin de donner un exemple concret je citerai mon département de langues romanes, avec 3000 étudiants dont 1700 étudiants de français, où nous disposons d'une chaire de littérature française francophone, une chaire de littérature française et espagnole, une chaire de littérature italienne et française et deux chaires de linguistique romane (la première diachronique et la seconde synchronique). À cela s'ajoute une douzaine d'enseignants non-habilités.

En raison de leur nombre limité, les professeurs sont certes des spécialistes dans certains domaines mais sont en même temps tenus à enseigner toute l'étendue des littératures et de la linguistique romanes. Ainsi, un professeur allemand peut être spécialiste de Pétrarque, de Zola et de Manzoni - notre système ignore cependant des profils de poste à la française du style «dix-huitièmiste». À cela s'ajoute la question (extrêmement délicate) de la langue. Traditionnellement les cours de littérature et de linguistique sont - au grand dam des étudiants - dispensés en allemand. Il y a à cela des raisons pratiques. Ainsi dans les petits départements un cours s'adresse à des étudiants de deux langues (un exemple classique d'un cours à double valence serait «L'écriture de la Shoah en France et en Italie: David Rousset et Primo Levi»). Mais il y a également des raisons historiques. La question du passé de la Romanistique allemande est encombrante et douloureuse et son rôle pendant le nazisme est loin d'être glorieux. Ainsi un certain nombre de professeurs juifs furent évincés de leurs chaires pendant le nazisme et réduits à l'exil (Auerbach, Spitzer), ou au désœuvrement humiliant (Klemperer). En ayant glorifié les exilés et en les célébrant comme la conscience morale de la Romanistique allemande après la Libération, on occulte une vérité qui dérange : l'adhésion massive des professeurs allemands au nazisme.

Comment cette adhésion à un régime francophobe peut-elle être expliquée ? En effet, après son institutionnalisation en 1830, la Romanistique devint une arme "antiromane » et cherchait à cimenter la suprématie allemande sur les pays «latins». Ces tendances revanchistes se sont cimentées dans les années 1910 et 1920 - notamment après la défaite de l'Allemagne en 1918. Avec l'arrivée au pouvoir des nazis en 1933, les Études Romanes, déjà largement marginalisées, se sont mises au service du Troisième Reich. Il convient de noter également que l'expulsion des professeurs juifs a permis à des individus de talent moindre d'accéder à des postes importants (Hausmann 175315/Gumbrecht 5-38).

Pour quelles raisons ces faits sont-ils importants pour notre contexte ? Il me semble que la Romanistique allemande ne se soit jamais entièrement remise de cette époque. La fuite des cerveaux vers les États-Unis (et parmi eux les représentants les plus éminents de notre matière) a causé des dommages intellectuels durables et on peut se demander si la Romanistique telle qu'elle se présente à l'heure qu'il est en est vraiment sortie indemne. En raison de la double spécialisation et d'une certaine 
tradition qui toutes deux ne sont pourtant que rarement mises en cause et très rarement discutées (à l'exception notable de Stenzel 2004), la Romanistique allemande demeure très attachée à la langue allemande dans les échanges académiques. On comprendra aisément que cet attachement contribue à un isolement international de plus en plus pesant: qui lit une thèse écrite en allemand, qui participe à un colloque en allemand, même si pour des intervenants extérieurs, de plus en plus souvent, on lui concède l'utilisation de sa langue ? C'est ainsi que la Romanistique est souvent isolée sur le plan international et manque de visibilité à l'échelle extra-européenne - un fait qui est heureusement en train de changer.

\section{2) La langue française en Allemagne}

Avant de vraiment nous pencher sur le rôle que jouent les Études Francophones en Allemagne, attardons-nous sur le statut du français en République Fédérale. Le site Internet wikipédia, cité car il s'agit de la seule source disponible, dans son entrée Francophonie dénombre 200.000 francophones en Allemagne (dont 165.000 expatriés de langue maternelle française), un chiffre qui paraît excessivement bas. Si effectivement l'entrée allemande Franఇösischunterricht (enseignement du français) fait remarquer à juste titre que le français, en dépit du nombre considérable d'élèves, n'est que relativement peu parlé en Allemagne, le chiffre toutefois de 35.000 locuteurs allemands me parait irréaliste, compte tenu du nombre d'étudiants qui sont issus de familles bilingues, ou qui ont étudié en France ou dans un autre pays francophone, ou qui ont été scolarisés dans un lycée français en Allemagne (qui existent à Berlin, Hambourg, Düsseldorf, Sarrebruck, Stuttgart, Francfort, Cologne, Munich, et Fribourg), ou qui sont titulaires d'un baccalauréat franco-allemand proposé dans de nombreux établissement surtout, mais non exclusivement, le long de la frontière avec la France.

L'Office Fédéral des Statistiques a publié le tableau suivant indiquant le nombre d'élèves allemands du secondaire et les langues étrangères qu'ils apprennent :

\begin{tabular}{|c|c|c|c|}
\hline \multirow{3}{*}{ Langues } & \multicolumn{3}{|c|}{ Année scolaire } \\
\hline & $2008 / 09$ & $2009 / 10$ & \\
\hline & absolu & Absolu & $\begin{array}{l}\text { Variations } \\
\text { en } \%\end{array}$ \\
\hline
\end{tabular}

\begin{tabular}{|l|l|l|l|}
\hline Anglais & 7309969 & 7480252 & $+2,3$ \\
\hline Français & 1700116 & 1694173 & $-0,3$ \\
\hline Latin & 832891 & 822673 & $-1,2$ \\
\hline Grec ancien & 15659 & 14385 & $-8,1$ \\
\hline Espagnol & 320599 & 337294 & $+5,2$ \\
\hline
\end{tabular}

(Statistisches Bundesamt 2011') 
Comme le tableau l'indique clairement, la situation du français en Allemagne reste confortable et sa baisse est relativement légère. Effectivement, la concurrence de l'espagnol souvent conjurée, reste relativement modérée, même si effectivement sa croissance est importante.

Or, contrairement au français, l'espagnol est considéré comme une langue plus facile et plus utile. Effectivement, ce dont souffre le français, est sa perception comme langue uniquement européenne et non pas comme langue internationale. Si, depuis la fin de la Seconde Guerre Mondiale, et c'est heureux, un fin réseau de collaborations entre la France et l'Allemagne s'est tissé, celui-ci semble limité à monopoliser la visibilité du français au seul contexte européen. La tentative de vouloir établir des cursus communs avec le Canada, par exemple, soulève une double difficulté : les frais d'inscription à l'Université canadienne nous semblent exorbitants (l'enseignement supérieur en Allemagne est totalement gratuit) et le manque de mobilité des étudiants canadiens, moins enclins à passer une ou deux années universitaires en Europe. Par ailleurs, s'il existe un réseau développé d'Instituts français en Allemagne, ceux-ci ne commencent que lentement à s'ouvrir à des questions qui dépassent le cadre francofrançais. C'est ainsi que le français en Allemagne continue à être perçu comme une langue essentiellement européenne ${ }^{1}$.

Après avoir présenté les prémisses institutionnelles et culturelles qui façonnent les modalités de fonctionnement de la Romanistique allemande, essayons d'établir un état des lieux des Études Francophones en Allemagne; afin de garder un minimum de distance néanmoins, nous nous contenterons de l'année 2009. A cet effet, nous consulterons dans un premier temps le Romanistisches Jabrbuch de ladite année. Celui-ci répertorie non seulement tous les professeurs (titulaires de chaire, bien entendu) mais encore toutes les thèses de doctorat et d'habilitation soutenues et un certain nombre de thèses en cours.

Les résultats de notre petite enquête sont effectivement assez surprenants: aucune thèse d'habilitation n'est consacrée à un thème relevant des Études Francophones, cependant un certain nombre de thèses de doctorat se penchent sur des problématiques francophones. Outre une thèse sur la chanson québécoise entre 1760 et 1840, furent soutenues des thèses de doctorat consacrés à Toussaint Louverture, trois thèses de doctorat sur la littérature francophone essentiellement d'Afrique Noire sont en cours à Bayreuth, ainsi qu'une thèse en cours sur la littérature des Caraỉbes et des questions de genre, sans que cependant le domaine linguistique analysé fût spécifié. On remarquera néanmoins que la grande majorité des thèses soutenues ou en cours sont consacrées à des sujets que l'on pourrait qualifier de franco-français (Bilanz 15-28). On ne peut pas parler de la déferlante «francophoniste» que quelques détracteurs - ces mêmes professeurs qui considèrent les Études Francophones comme une menace majeure pour la Romanistique allemande traditionnelle - voient venir.

1 On trouvera des propositions pour remédier à ce dilemme tout à fait intéressant par ailleurs dans Kolboom 1999. Concernant la diffusion de la notion d'un savoir sur la Francophonie en Allemagne, on consultera avec le plus grand profit Kolboom 2002, Kolboom et al. 2002. 
Également en 2009 s'est tenu le «Romanistentag », la réunion biannuelle de tous les romanistes allemands, qui alterne avec la réunion biannuelle des associations consacrées aux langues respectives de la « Romania ». Ainsi, se tiennent en 2010 se sont tenues le Franko-Romanistentag, le Italianistentag etc., c'est-à dire les conférences consacrées aux différentes langues romanes. Le Romanistentag s'organise par «ateliers », en allemand "Sektionen » consacrés à un sujet précis de la Romanistique qui collent plus ou moins avec un sujet général, un sujet « cadre ». Présentons quelques-uns des intitulés de ces sections, qui sont extrêmement instructifs quant à l'imaginaire de la Romanistique allemande. Voici quelques exemples de sections de notre domaine de recherche, la littérature :

II.1 - Der Petrarkismus: ein europäischer Gründungsmythos

Le petrarquisme, un mythe fondateur européen

II.2 - Der europäische Roman um 1800

Le roman européen autour de 1800

II.4 - Traumwissen und Traumpoetik in Frankreich, Italien und Deutschland seit 1800

Le savoir et la poétique oniriques en France, Italie et Allemagne depuis 1800

II.5 - Autobiographie und Kultur: Diversität pragmatischer Einbettung einer Grenzgattung in der Romania

L'autobiographie et la culture - diversités pragmatiques d'un genre-limite dans la Romania

II.6 - Reflexe eines Umwelt- und Klimabewusstseins in fiktionalen Texten der Romania Consciences écologiques et climatiques dans quelques textes fictionnels de la Romania

III. Kultur- und Medienwissenschaft

III.1 - Die Moralischen Wochenschriften in der Romania - eine paneuropäische Gattung Les hebdomadaires moralistes de la Romania - un genre paneuropéen

III.4 - ,Nation’ und ,Region’ - Zur Aktualität intrakultureller Prozesse in der globalen Romania Nation et région - Actualités des processus intraculturels dans la Romania mondiale

III.5 - Transatlantische Perspektiven in der Romanistik: Das kulturelle Feld in der Karibik Romanistique et perspectives transatlantiques - Le champ culturel des Caraibes (Romanistentag)

Ces thématiques sont à chaque fois relativement larges afin de permettre au plus grand nombre et aux diverses langues d'y trouver leur place. Si les Caraibes constituent effectivement un champ d'investigation qui s'inscrit dans les Études Francophones, les autres ateliers peuvent très bien accueillir des interventions sur la Francophonie sans toutefois en constituer le centre.

La différence entre la Romanistique générale et la Franco-Romanistique se fait jour lorsqu'on consulte le programme de la Conférence nationale des franco-romanistes en 2010. Présentons une sélection des ateliers proposés :

8. Littératures indianocéaniques: regards déracinés périphériques sur les espaces culturels et urbains dans les littératures francophones de l'océan indien de l'ouest

9. Figurations de la ville-palimpseste 
10. Scénarios de l'espace - Littérature, film et techniques de déplacement dans l'espace urbain

14. La cour et la ville: Pratique sociale et esthétique sous l'Ancien Régime : Entre courtoisie et honnêteté (1630-1750)

16. Espaces (post)coloniaux et gender

22. Bruxelles surréaliste. Topographies d’une cité (Romanistentag)

On retiendra d'un côté la plus forte présence de la langue française (un fait d'ailleurs que les puristes reprochent à la ce genre de manifestations) mais également la plus forte représentation de toutes les questions relevant des Études Postcoloniales et Francophones. On peut donc affirmer que les Études Francophones percent lentement mais demeurent néanmoins fortement minoritaires. Elles ont cependant réussi à s'imposer au fil des vingt dernières années.

Il convient à présent de s'interroger sur les vecteurs qui ont permis aux Études Francophones de percer. Relativement faible sur le plan institutionnel, elles sont surtout le fait de certains professeurs qui, dès les années 1980, ont reconnu l'importance de ce domaine de recherche et ont pris des initiatives en faveur de l'implantation des Études Francophones en Allemagne. Dans un environnement encore plus réactionnaire qu'aujourd'hui, le courage de formuler, dès 1988, des phrases comme celles que l'on va citer, est difficilement explicable aujourd'hui :

«Glücklicherweise für die Fortune dieser Autoren (unglücklicherweise für die gesellschaftliche Bedeutung des Faches Romanistik) in der Bundesrepublik warten der Buchmarkt und das lesende Publikum gar nicht erst auf die Mittlerdienste der literaturwissenschaftlichen Romanistik. Im Falle der Karibik wie im Falle der Vermittlung afrikanischer Literatur in französischer Sprache kamen und kommen die wichtigsten Anstösse zur Beschäftigung mit diesen Autoren von Journalisten und Kritikern außerhalb des universitären Milieus. [...] Die gelegentlichen Sondernummern romanistischer literaturwissenschaftlicher Zeitschriften sind zwar begrüßenswert, weil sie von Zeit zu Zeit in gebündelter Form eine Art Bestandaufnahme bringen, aber sie erfüllen letztlich doch nur eine Art Alibi-Funktion, wenn man bedenkt, dass es in Frankreich, Kanada und den USA schon lange spezialisierte Zeitschriften zu diesen Literaturen gibt, dass auf den Kongressen der «Associsation Internationale de Littérature Comparée » schon seit langem Sektionen für afrikanische und karibische, wie generell «neue » Literaturen bestehen, dass zu einzelnen Autoren wie Anne Hébert aus dem frankophonen Qebec (nach einer Bibliographie von 1984) zehn Buchpublikationen, über 40 Hoschschulschriften und über 100 Zeitschriftenaufsätze vorliegen, aber nichts davon in der Bundesrepublik und in deutscher Sprache erschienen ist $»^{2}$. (Riesz 106-7)

\footnotetext{
2 Heureusement pour la fortune de ces auteurs (et malheureusement pour l'importance de la Romanistique dans la société de la République fédérale) le marché du livre et le public n'attendent pas que la Romanistique fasse son travail de médiation. Pour ce qui est des Caraïbes et de l'Afrique francophone, ce sont les journalistes et les critiques littéraires non-universitaires qui ont été décisifs dans la diffusion de ces auteurs. Les numéros spéciaux des revues méritent certes notre attention et notre soutien, puisqu'ils fournissent, de manière condensée un état des lieux, mais finalement ils font figure d'alibi quand on tient compte des faits suivants: Aux congrès de L'Association Internationale de Littérature Comparée il existe des ateliers sur les littératures africaines et des Caraibes ainsi que les littératures nouvelles depuis fort longtemps. Par ailleurs, il y a sur des écrivains comme la Québécoise Anne Hébert (d'après une bibliographie de 1984) dix publications sous formes de livre, 40 publications universitaires et une centaine d'articles, mais rien n'a paru en République fédérale ni en langue allemande (La traduction est de l'auteur).
} 
Dans un atelier parallèle, consacré à la canonisation, on pouvait lire ces phrases non moins courageuses :

«Nicht nur in Dakar, Rio de Janeiro und Montréal, sondern auch in der bundesrepublikanischen Romanistik sollte - beispielsweise - den Werken eines Alejo Carpentier, einer Maryse Condé ode reines Ousame Sembène mindestens die gleiche Aufmerksamkeit schenken wie den in Forschung und Lehre zweifelsohne überrepräsentierten Avantgarde-Strömungen des 21. Jahrhunderts vom Surrealismus bis zum Nouveau nouveau Roman $»^{3}$. (Berger/Lüsebrink 189)

Le présent texte ne cherche absolument pas à procéder à une sorte d' «hagiographie » de la Romanistique allemande. Or il convient de souligner que ce sont quelques rares personnes - contre des résistances puissantes et institutionnelles -qui ont travaillé pour la solide implantation des Études Francophones en Allemagne. Ce fait néanmoins souligne également la fragilité, lorsqu'un des "hérauts » de la Francophonie en Allemagne part à la retraite, il n'est pas du tout certain que son successeur suive l'orientation de ses prédécesseurs. Faibles sur le plan institutionnel, les Études Francophones paraissent assez fragilisées dans la mesure où son implantation et son succès tient davantage à des profils personnels. À cela s'ajoute la faiblesse au niveau des masters. Si l'université allemande vit très, très mal la réforme " de Bologne » ${ }^{4}$, cette dernière n'a pas non plus contribué au rayonnement des Études Francophones. Il existe à présent seulement deux masters qui portent dans leur dénomination le mot «francophone », le premier à Bayreuth (Études Francophones), le deuxième à Leipzig (Internationale Frankreich- und Frankophonie-Studien), ce qui n'empêche habituellement pas les étudiants de choisir une thématique francophone pour leurs mémoires de master dans une filière «classique » de Romanistique.

Vu l'orientation littéraire de l'auteur de ces lignes, nous avons un peu délaissé la linguistique. Il convient toutefois de souligner que ce sont aussi les linguistes, peut-être avec moins d'angoisse et d'arrogance que les littéraires qui se sont penchés sur des questions qui relèvent des Études Francophones. Ce sont notamment des études de terrain, mais aussi des thèmes dans le domaine de la linguistique du contact et du plurilinguisme qui sont traités par de nombreux chercheurs allemands. Il est bon cependant de ne pas occulter le fait que de plus en plus de linguistes allemands publient leurs textes... en anglais. Effectivement l'anglais est en passe de devenir la langue d'échange académique pour de nombreux linguistes. On peut se poser la question de savoir si cette tendance (que l'auteur de ces lignes trouve fâcheuse) n'est pas le résultat finalement du maintien désespéré de la langue allemande: à défaut d'envisager le français comme une langue internationale, on passe de l'allemand (langue nationale... quoique...) à l'anglais (langue internationale). Cependant, cette tendance ne peut pas non plus escamoter le fait que l'ouvrage de référence en langue allemande sur la Francophonie a été rédigé par un linguiste (Erfurt 2005).

\footnotetext{
${ }^{3}$ Ce n'est pas qu’à Dakar, à Rio de Janeiro ou à Montréal, mais aussi dans la Romanistique allemande que l'on devrait prêter la même attention aux œuvres - par exemple - d'Alejo Carpentier, de Maryse Condé ou de Ousmane Sembène - qu'aux mouvements surreprésentés dans l'enseignement et la recherche comme le surréalisme ou le Nouveau nouveau roman.

${ }^{4}$ Le processus de Bologne fur initié en 1999 par les ministres de l'Éducation de l'Europe. Il prévoit une standardisation des diplômes et de l'organisation des études. Souvent réalisé hâtivement, le processus de Bologne a entraîné des manifestations massives en Allemagne et en Autriche, car il fut envisagé comme l'avènement de l'idéologie néolibérale dans les universités.
} 
Il convient de noter par ailleurs que les Études Culturelles, souvent liées aux Études Francophones, peinent à s'imposer en Allemagne - une situation néanmoins qui est en train de changer. Déjà en 1985, on discutait âprement de l'avenir de la "civilisation française » au sein de la Romanistique allemande. Si en 1985, on se disputait sur le passage de la "Landeskunde » (terme que l'on peut traduire de manière approximative par " civilisation») vers la «Landeswissenschaft». (terme que l'on peut traduire de manière aussi approximative par "science du pays»), le centre du débat tournait autour de la question de savoir si la «Kulturwissenschaft» (sciences de la culture, l'équivalent allemand des Cultural Studies d'inspiration anglo-saxonne) constituent un champ de recherche autonome à même titre que la littérature et la linguistique, ou si elle met à la disposition des étudiants un savoir "utile » puisque en effet ceux-ci sont dans leur écrasante majorité de futurs enseignants (ainsi dans mon Université plus de $80 \%$ des étudiants sont inscrits dans la filière dite en novlangue «bolognese » Bachelor of Education).

Il est à noter également que les Études francophones ne sont pas l'apanage des romanistes : il existe également de nombreux sociologues, politologues et historiens, des spécialistes de la culture française qui se penchent sur la question de manière plus ou moins engagée. Ce sont eux aussi qui font vivre et qui propagent cette réalité francophone méconnue en Allemagne. Il est à souligner néanmoins que la Romanistique allemande, à l'heure qu'il est, connaît des changements importants. Nous sommes en train de passer de la Romanistique allemande «vieux jeu » à une vision beaucoup plus moderne de la Romanistique dans laquelle les Études Francophones comme les autres domaines d'étude ont toute leur part. Si effectivement la langue française est aujourd'hui en crise, cette dernière amène à réfléchir à de nouveaux modes de diffusion et d'enseignement et est donc susceptible de faire ainsi' rebondir sur de nouvelles bases l'enseignement des Lettres Francophones en Allemagne.

\section{3) En guise de conclusion}

La première difficulté de ce texte résidait bien évidemment dans le fait que les Études Francophones se situent largement en marge des Études Romanes en Allemagne. Ce fait s'explique par l'histoire complexe de la Romanistique allemande et par son évolution. Nous avons pu constater également que l'histoire des relations franco-allemandes et la réconciliation franco-allemande après la guerre (une chose en soi parfaitement heureuse) et les nombreuses initiatives franco-allemandes qui en découlent tendent quelque peu à occulter le fait que le français est aussi une langue internationale et une langue parlée autre part qu'en France et en Europe.

Les Études Francophones faibles sur le plan institutionnel ont réussi à s'imposer malgré tout en Allemagne en raison de l'engagement d'un certain nombre de professeurs, souvent contre de puissantes résistances institutionnelles. Force est de noter néanmoins que ce combat est loin d'être gagné. On pourrait citer, à titre d'exemple, la fermeture prévue de la seule chaire allemande uniquement consacrée aux Études Francophones et françaises dans une perspective des Cultural Studies à Dresde. Extrêmement performante et active dans la diffusion d'un savoir sur une réalité méconnue en Allemagne, cette chaire sera supprimée lors du départ à la retraite du titulaire actuel et transformée en chaire «français/italien - littérature et culture ». La 
Francophonie serait-elle une réalité trop coûteuse pour ce pays voisin de la France? En même temps, il y a une réelle demande de Francophonie de la part des étudiants de français, nombreux dans notre pays. On est donc en droit d'espérer des changements pour les années à venir. 


\section{Bibliographie}

Berger, Günther/Lüsebrink, Hans-Jürge. «Kanonbildung in der Romania », Nies, Fritz/Grimm, Reinhard, Ein "unmögliches Fach»: Bilanz und Perspektiven der Romanistik. Tübingen: narr, 1985.

"Bilanz 2009". Romanistisches Jabrbuch. Berlin: de Gruyter, 2010.

Christmann, Hans Helmut. Romanistik und Anglistike an der deutschen Universität im 19. Jabrbundert, Mainz: Akademie der Wissenschaften und der Literatur, 1985.

Deutscher Frankoromanistenverband. Frankoromanistentag 2009, site web, 29 mai 2011.

Deutscher Romanistenverband. Romanistentag 2010, site web, 29 mai 2011.

Erfurt, Jürgen. Frankophonie. Sprache-Diskurs-Politik. Tübingen: Francke, 2005.

Gumbrecht, Hans-Ulrich. Vom Leben und Sterben der großen Romanisten, München: Hanser, 2002.

Hausmann, Frank-Rutger. «Vom Strudel der Ereignisse verschlungen ». Deutsche Romanistik im Dritten Reich, Frankfurt am Main: Klostermann (Analecta Romanica), 2000.

Kolboom, Ingo. «Quelle approche pour la Francophonie? Plaidoyer pour une réflexion allemande et franco-allemande ». Grenzgänge 6.12 (1999): 169-182.

Kolboom, Ingo. «Francophonie: Von der kulturellen zur politischen Frankophonie »,

Kolboom, Ingo et al., Handbuch Franæösisch. Sprache, Literatur, Kultur und Geschicbte, Berlin: Erich Schmidt Verlag, 2002.

Riesz, Janos. «Frankophone Literaturen außerhalb Europas» Nies, Fritz/Grimm, Reinhard, Ein « unmögliches Fach »: Bilan₹. und Perspektiven der Romanistik. Tübingen: narr, 1985.

Statistisches Bundesamt. Zabl der Schülerinnen und Schüler im Fremdsprachenunterricht, site web, 12 avril 2011.

Stenzel, Harald. "Literaturwissenschaft - Landeskunde - Kulturwissenschaft, oder die verdrängten Probleme eines hybriden Fachs. Anmerkungen zur Situation der

Romanistik", Nünning, Ansgar/Sommer, Roy. Kulturwissenschaftliche Literaturwissenschaft. Tübingen: narr, 2004.

Wikipedia (en français), « Francophonie », site web, 14 mai 2011.

Wikipedia (en allemand), « Französischunterricht», site web, 14 mai 2011. 